\title{
Astashine Capsules: A Natural Immune Booster Enhances Natural Immunity
}

\author{
Govind Shukla, Uddhav L Kanade, Monica Yadav, M.Sabitha \& C.J.Sampath Kumar
}

Lactonova Nutripharm (P) Ltd., Makers of ASTASHINE Capsules, 81/3, IDA Mallapur, Hyderabad, Telangana, India-500 076.

DOI: $10.46382 / M J B A S .2020 .4203$

\section{ABSTRACT}

Astaxanthin is a naturally occurring carotenoid which is derived from the microalgae Haematococcuspluvialis. As well as being the most powerful antioxidant known to science, it also has potent anti-inflammatory properties. Naturalastaxanthin's distinct advantage in comparison to other antioxidants, is its ability to span the entire lipid bilayer of the cell membrane, thus providing superior protection from the inside out. Natural astaxanthin has a strong ability to both balance and strengthen the immune system. This article reviews the current available scientific literature regarding the effect of astaxanthin from the algae Haematoccus pluvialis in Astashine capsules as a natural immune booster.

Keywords: Astashine Capsules, Natural Immune Booster.

\section{Introduction}

The immune system is made up of a network of cells, tissues and organs that work together to protect the body against infectious microorganisms, such as certain bacteria and viruses; whilst also working to destroy any infectious microorganisms that manage to invade the body.

Phagocytes are cells that destroy invading organisms, while lymphocytes are cells that allow the body to remember and recognize previous invaders and help the body destroy them - the innate immune response.

Natural astaxanthin has shown positive effects and substantial benefit in enhancing the capacity of both lymphocytes and phagocytes.

\section{Astashine capsules as a potent Immune Agent}

Inflammation is the body's biological response to harmful stimuli. Acute inflammation is the initial response to such stimuli and in most cases it can be visually identified. The other form of inflammation is "chronic inflammation";

It is often called the silent killer since there are very few, if any, symptoms of this type of inflammation. Unlike the acute type, chronic inflammation builds up in the body as a result of the immune system constantly responding to threats. Chronic inflammation is one of the major causes of accelerated aging and many of its associated diseases such as chronic heart disease, arthritis or allergies.

It is characterized by the release of cytokines and pro-inflammatory markers such as tumor necrosis factor- $-\alpha$ (TNF- - $\alpha$ ), C-reactive protein (CRP) and others. Luckily, we can test for inflammation by measuring these different biological markers in the blood which will give an indication of inflammation levels. Natural astaxanthin has been shown to help significantly decrease the expression of pro-inflammatory markers and mediators, thus providing potent anti-inflammatory protection in the body. Several studies have been conducted to understand the anti-inflammatory mechanism of astaxanthin and it is thought to be related to the inhibition of the Nf-kB inflammatory pathway [5]. 


\section{Composition of Astashine capsules}

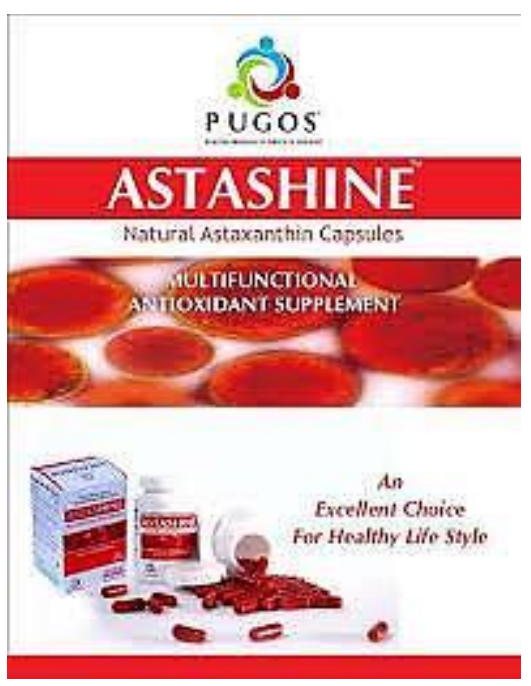

Astaxanthin - 2mg

(Naturally derived from Haematococcus pulvialis algae extract, which is microencapsulated)

\section{Mechanism of Action}

Astaxanthin's outstanding properties as an anti-inflammatory also play a major role in its ability to enhance immune function. Due to the multitude of ways in which Astaxanthin combats inflammation, it is a very special anti-inflammatory indeed. Both in-vitro and in-vivo research has been done to uncover its mechanism of action as an anti-inflammatory. This mechanism has been further demonstrated in several double blind, placebo controlled human clinical trials on various inflammatory conditions. Astaxanthin works to suppress different inflammatory mediators. Among these mediators are tumor necrosis factor alpha (TNF-a), prostaglandin E-2 (PGE-2), interleukin 1B (IL-1b) and nitric oxide (NO). In one experiment done with mice and also in-vitro, Astaxanthin was shown to suppress TNF-a, PGE-2, IL-1b, NO as well as the Cox-2 enzyme and nuclear factor kappa-B [1-4].

Another study done the same year was led by a researcher from Japan's Hokkaido University Graduate School of Medicine. Here, the researchers found similar results: Astaxanthin was shown in vitro to decrease the production of NO, PGE-2 and TNF-a. This study also looked at Astaxanthin's anti-inflammatory effect in the eyes of rats. The researchers induced uveitis (inflammation of the inner eye including the iris) and found that Astaxanthin had a "dose dependent ocular anti-inflammatory effect, by the suppression of NO, PGE-2 and TNF-a production, through directly blocking nitric oxide synthase enzyme activity" [2].

\section{Clinical Study Reports of Astashine Capsules}

\section{Astashine capsules enhances Immune Response}

The immune system is made up of a network of cells, tissues and organs that work together to protect the body against infectious microorganisms, such as certain bacteria and viruses; whilst also working to destroy any infectious microorganisms that manage to invade the body. 
Phagocytes are cells that destroy invading organisms, while lymphocytes are cells that allow the body to remember and recognize previous invaders and help the body destroy them - the innate immune response.

Natural astaxanthin has shown positive effects and substantial benefit in enhancing the capacity of both lymphocytes and phagocytes. The first human study to show this effect was published by Park et al. in 2010. In this double blind, placebo controlled study, 42 individuals received 0 or $8 \mathrm{mg} / \mathrm{d}$ astaxanthin (AX) vs placebo. Lymphocyte count was significantly increased for the AXgroup [2].

In addition, those receiving $\mathrm{AX}$ demonstrated a significant increase in the levels of $\mathrm{B}$ and $\mathrm{T}$ cells (figure 1).

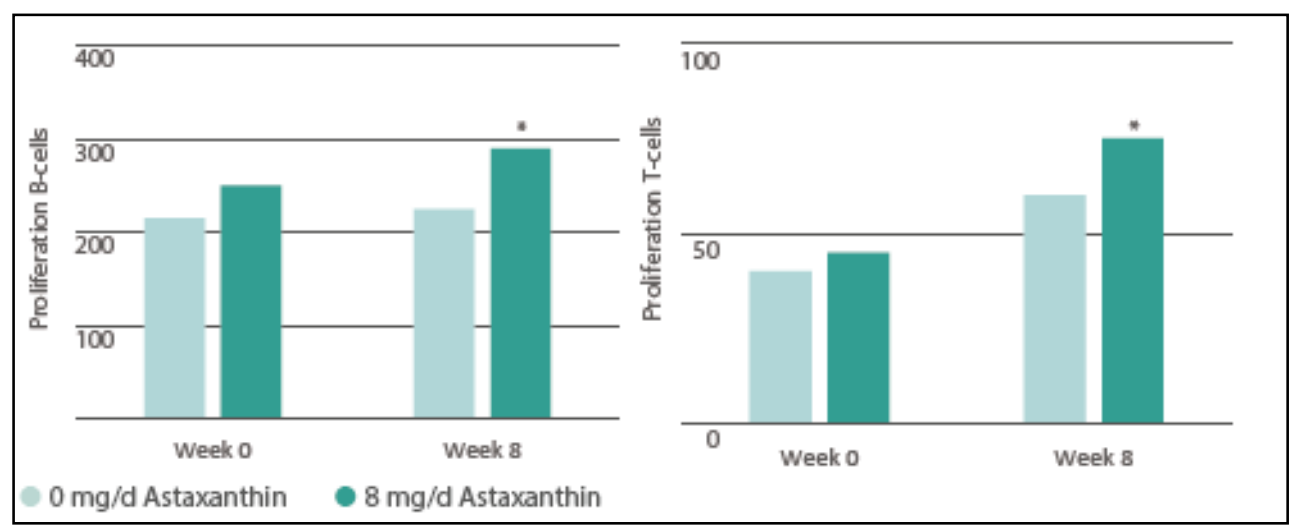

Fig.1. The effect of astaxanthin on immune response was measured in a double blind, randomized studyinvolving42 subjects. B-cells and T-cells were exposed to high concentrations of mitogens and their capacity to proliferate was measured (2). *p<0.05 compared with control
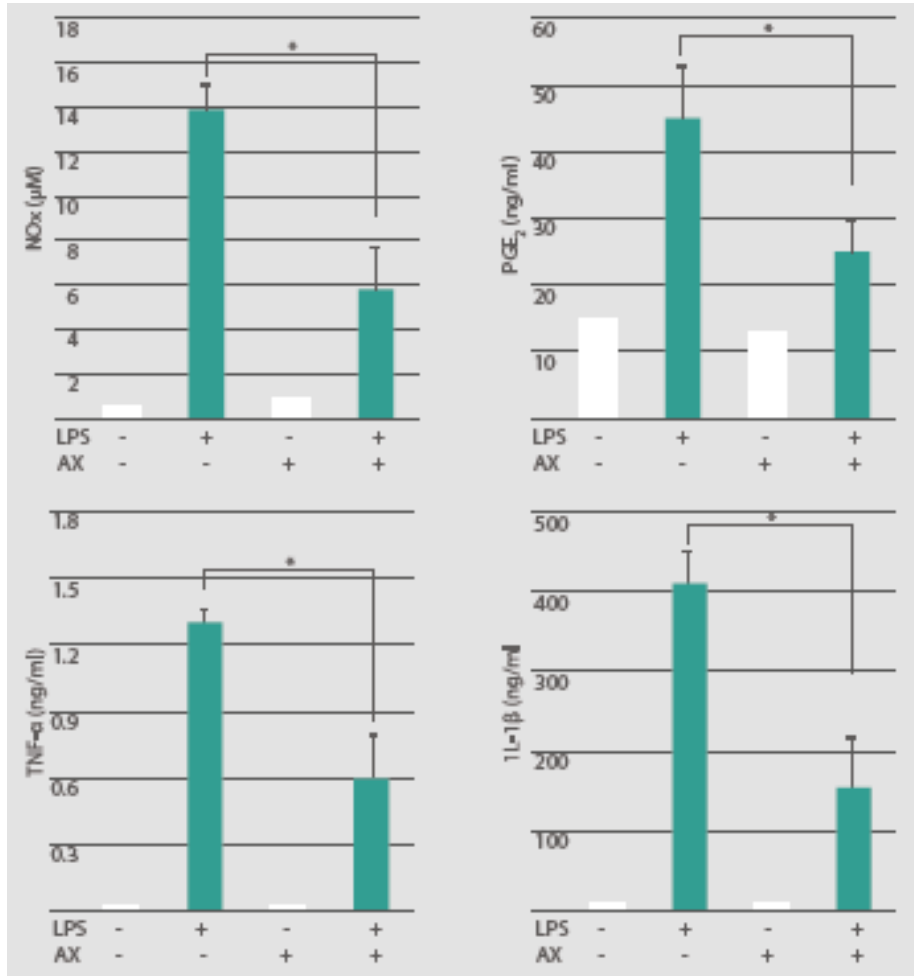

Fig.2. LPS-induced inflammation showing significant decrease in plasma levels of pro-inflammatory markers NO, $P G E 2, T N F-\alpha$, and IL-1 $\beta \quad$ LPS=Lipopolysaccharide AX=Astaxanthin 
Research has shown that natural astaxanthin is also a potent anti-inflammatory and is particularly effective against chronic inflammation. To test its efficacy, scientists have examined the levels of pro-inflammatory markers with and without the presence of astaxanthin. In a 2003 model study, Seon et al. found that astaxanthin significantly decreased the expression of different pro-inflammatory markers in Lipopolysaccharide-induced inflammation (LPS).

In another randomized, double blind placebo controlled study, Park et al. (2010) examined 42 subjects for the pro-inflammatory marker CRP. The astaxanthin group supplemented with $2 \mathrm{mg} / \mathrm{d}$ for 8 weeks showed that the levels of CRP were significantly reduced [2].

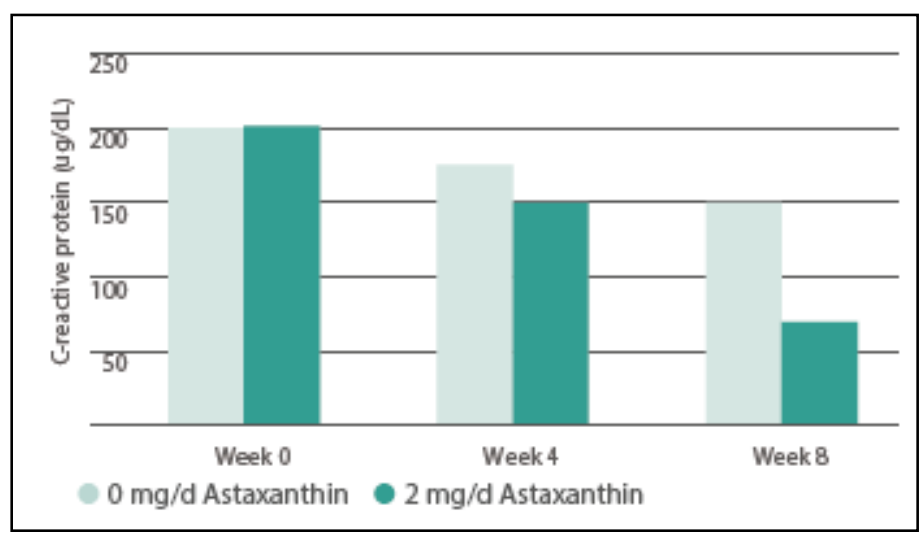

Fig.3. 8 weeks of supplementation with $2 \mathrm{mg} / \mathrm{d}$ natural astaxanthin significantly reduced the expression of the pro-inflammatory marker $C$-reactive protein (CRP)

\section{Safety of Astashine Capsules}

Astaxanthin has demonstrated safety in numerous human clinical trials. In one open-label clinical study on subjects with metabolic syndrome $(\mathrm{n}=17)$. Astaxanthin ( $16 \mathrm{mg} / \mathrm{day}$, for three months) significantly raised blood bilirubin $(\mathrm{p} \leq 0.05)$, potassium $(\mathrm{p} \leq 0.05)$, and creatine kinase $(\mathrm{p} \leq 0.01)$, although all three values remained within normal range. Also, astaxanthin significantly lowered the liver enzyme gamma-glutamyl transpeptidase (GGTP; $\leq \leq 0.05$ ). Since the researchers noted this enzyme was abnormally elevated in 11 of the 17 subjects at baseline, this astaxanthin effect may have been beneficial. Animal experiments have investigated astaxanthin at levels well over $120 \mathrm{mg} /$ day in human equivalents, without causing apparent harm. Hoffman-La Roche confirmed its safety with extensive tests, including acute toxicity, mutagenicity, teratogenicity, embryotoxicity, and reproductive toxicity.

\section{Suggested Dosage}

The doses of astaxanthin used in clinical trials have ranged from $1 \mathrm{mg}$ /day to $40 \mathrm{mg} /$ day (with the majority in the 6-12 mg range); single-dose pharmacokinetic studies used up to $100 \mathrm{mg}$ per dose. As a dietary supplement, astaxanthin should be taken along with fats, with or immediately prior to meals, to ensure its optimal absorption.

\section{Summary \& Conclusion}

Studies demonstrate that astaxanthin helps to balance the immune system by stimulating its infection. While also helping suppress the overactive immune responses that create needless inflammation [6]. Astaxanthin increases the 
numbers and activity of white blood cells called lymphocytes and natural killer cells that are responsible for creating the body's innate immune response to invaders [7-9].

Astaxanthin has similar immune-boosting effects in humans, improving the ability of protective white blood cells to surround and destroy infecting organisms, especially fungi such as Candida albicans. [10] Astaxanthin also protects human lymphocytes and neutrophils against the oxidant stresses imposed by the actions of certain white blood cells without reducing the killing effects of white blood cells themselves [11].

Human studies reveal astaxanthin's beneficial actions on immune system in patients with allergies and asthma. When astaxanthin was given to asthmatic patients, it suppressed reactive cell activation as well as or better than the antihistamine drugs [12].

\section{References}

1. Chew, B.P. et al.,2011. Dietary astaxanthin enhances immune response in dogs. Vet Immunol and lmmunopathol.140 (2011) 199-206.

2. Park, J.S., Chyun, J.H., Kim, Y.K., Line, LL., Chew, B.P., 2010.Astaxanthin decreased oxidative stress and inflammation and enhanced immune response in humans. Nutr. Metab.5, 7-18.

3. Park, J.S., Kim, H.W., Mathison, B.D., Hayek, M.G., Massimino, S., Reinhart, G.A., Chew, B.P., $2010 b$. Astaxanthin uptake in domestic dogs and cats. Nutr. Metab.7, 52-59.

4. Macedo RC et al., Astaxanthi $\mathrm{n}$ addition improves human neutrophils function: in vitro study. Eur $\mathrm{J}$ Nutr.2010;49:447- 457.

5. Seon-Jin L. et al. 2003. Astaxanthin Inhibits Nitric Oxide Production and Inflammatory Gene Expression by Suppressing IкB Kinase-dependent NF-кB Activation. Mol. Cells, Vol. 16, No. 1, pp. 97-105.

6. Chew BP, Park JS. Carotenoid action on the immune response. J Nutr. 2004 Jan;134(1):257S-61S.

7. Nakao R, Nelson OL, Park JS, Mathison BD, Thompson PA, Chew BP. Effect of dietary astaxanthin at different stages of mammary tumor initiation in BALB/c mice. Anticancer Res. 2010 Jun; 30(6):2171-5.

8.Chew BP, Mathison BD, Hayek MG, Massimino S, Reinhart GA, Park JS. Dietary astaxanthin enhances immune response in dogs. Vet Immunol Immunopathol. 2011 Apr 15;140(3-4):199-206.

9.Park JS, Mathison BD, Hayek MG, Massimino S, Reinhart GA, Chew BP. Astaxanthin stimulates cell-mediated and humoral immune responses in cats. Vet Immunol Immunopathol. 2011 Dec 15;144(3-4):455-61.

10.Macedo RC, Bolin AP, Marin DP, Otton R. Astaxanthin addition improves human neutrophils function: in vitro study. Eur J Nutr. 2010 Dec;49(8):447-57.

11.Bolin AP, Guerra BA, Nascimento SJ, Otton R. Changes in lymphocyte oxidant/antioxidant parameters after carbonyl and antioxidant exposure. Int Immunopharmacol. 2012 Dec;14(4): 690-7.

12. Mahmoud FF, Haines DD, Abul HT, Abal AT, Onadeko BO, Wise JA. In vitro effects of astaxanthin combined with ginkgolide B on T lymphocyte activation in peripheral blood mononuclear cells from asthmatic subjects. $J$ Pharmacol Sci. 2004 Feb;94(2):129-36. 